\section{(2) OPEN ACCESS}

\title{
Limit of detection of troponin discharge strategy versus usual care: randomised controlled trial
}

\author{
Edward Watts Carlton (1) , Jenny Ingram, ${ }^{2}$ Hazel Taylor, ${ }^{3}$ Joel Glynn, ${ }^{2}$ \\ Rebecca Kandiyali, ${ }^{2}$ Sarah Campbell, ${ }^{4}$ Lucy Beasant, ${ }_{1}^{2}$ Shahid Aziz, ${ }^{5}$ Peter Beresford, ${ }^{6}$ \\ Jason Kendall, ${ }^{1}$ Adam Reuben, ${ }^{7}$ Jason E Smith, ${ }_{1,9}^{8,9}$ Rebecca Chapman, ${ }^{4}$ \\ Siobhan Creanor, ${ }^{10}$ Jonathan Richard Benger ${ }^{11,12}$
}

- Additional material is published online only. To view please visit the journal online (http://dx.doi.org/10.1136/ heartjnl-2020-316692).

For numbered affiliations see end of article.

Correspondence to Dr Edward Watts Carlton, Emergency Department, North Bristol NHS Trust, Bristol, BS10 5NB, UK:

eddcarlton@gmail.com

Received 5 February 2020 Revised 27 March 2020 Accepted 30 March 2020 Published Online First 5 May 2020

\section{SLinked}

- http://dx.doi.org/10.1136/ heartjnl-2020-317012

Check for updates

(c) Author(s) (or their employer(s)) 2020. Re-use permitted under CC BY-NC. No commercial re-use. See rights and permissions. Published by BMJ.

To cite: Carlton EW, Ingram J, Taylor H, et al. Heart 2020;106:1586-1594.

\section{ABSTRACT}

Introduction The clinical effectiveness of a 'rule-out' acute coronary syndrome (ACS) strategy for emergency department patients with chest pain, incorporating a single undetectable high-sensitivity cardiac troponin (hs-cTn) taken at presentation, together with a nonischaemic ECG, remains unknown.

Methods A randomised controlled trial, across eight hospitals in the UK, aimed to establish the clinical effectiveness of an undetectable hs-cTn and ECG (limit of detection and ECG discharge (LoDED)) discharge strategy. Eligible adult patients presented with chest pain; the treating clinician intended to perform investigations to rule out an ACS; the initial ECG was non-ischaemic; and peak symptoms occurred $<6$ hours previously. Participants were randomised 1:1 to either the LoDED strategy or the usual rule-out strategy. The primary outcome was discharge from the hospital within 4 hours of arrival, without a major adverse cardiac event (MACE) within 30 days.

Results Between June 2018 and March 2019, 632 patients were randomised; 3 were later withdrawn. of 629 patients (age 53.8 (SD 16.1) years, 41\% women), $7 \%$ had a MACE within 30 days. For the LoDED strategy, 141 of 309 (46\%) patients were discharged within 4 hours, without MACE within 30 days, and for usual care, 114 of 311 (37\%); pooled adjusted OR 1.58 (95\% $\mathrm{Cl} 0.84$ to 2.98). No patient with an initial undetectable hs-cTn had a MACE within 30 days.

Conclusion The LODED strategy facilitates safe early discharge in $>40 \%$ of patients with chest pain. Clinical effectiveness is variable when compared with existing rule-out strategies and influenced by wider system factors.

Trial registration number ISRCTN86184521.

\section{INTRODUCTION}

The number of patients attending emergency departments (EDs) worldwide continues to rise. Across England and Wales, there were over 23 million attendances in 2017-2018, a rise of over $20 \%$ in a decade. ${ }^{1}$ Chest pain makes up $6 \%$ of ED attendances in the UK and is the most common reason for emergency hospital admission. ${ }^{2}$ Many patients with chest pain have prolonged hospital stays during which they undergo testing to rule out acute myocardial infarction (AMI), yet $90 \%$ of patients are found to have a non-cardiac cause of chest pain. ${ }^{2}$ Prolonged assessment leads to increased costs and ED crowding. ${ }^{3}$

For patients with suspected cardiac chest pain, the diagnostic biomarker of choice is cardiac troponin, a marker of myocardial necrosis. ${ }^{4}$ Highsensitivity cardiac troponin (hs-cTn) assays have been developed, and very low concentrations can be measured. ${ }^{5}$ This has led to improved diagnostic accuracy earlier after chest pain onset and development of rapid 'rule-out' strategies for low-risk patients. ${ }^{6-15}$ These strategies have predominantly been evaluated within observational cohorts, and a lack of high-quality interventional trial data means that the potential of hs-cTn assays to improve clinical effectiveness in the rapid discharge of low-risk patients with chest pain admitted to the ED remains poorly understood. ${ }^{14}$

A rapid rule-out strategy incorporating a single undetectable (below the limit of detection (LoD)) hs-cTn taken at ED presentation, together with a normal ECG, has been extensively evaluated in observational studies. ${ }^{8-15}$ While this limit of detection and ECG discharge (LoDED) strategy maintains a high diagnostic accuracy for both AMI and major adverse cardiac events (MACEs), across populations, the proportion of patients actually discharged after a single blood test, and consequently its clinical and cost effectiveness, remains unknown. ${ }^{3} 14$ Due to concerns about reporting imprecision, the undetectable cut-off has not been approved for use by the US Food and Drug Administration, ${ }^{16}$ while in Europe, consensus guidelines include caveats to its clinical use. European Society of Cardiology (ESC) guidelines recommend that the undetectable cut-off is only used if blood sampling occurs more than 3 hours after chest pain onset, and UK National Institute of Health and Care Excellence guidelines suggests it should be confined to low-risk patients identified using a validated risk score. ${ }^{1718}$ This may explain why around only $3 \%$ of hospitals within the UK use the undetectable cut-off in clinical practice. ${ }^{19}$

We aimed to determine whether the LoDED strategy, when used irrespective of chest pain onset time, without risk scores and across different hs-cTn assays, would increase the rate of safe early discharge from hospital in patients with suspected cardiac chest pain, when embedded within routine ED practice, and without an increase in healthcare costs. 


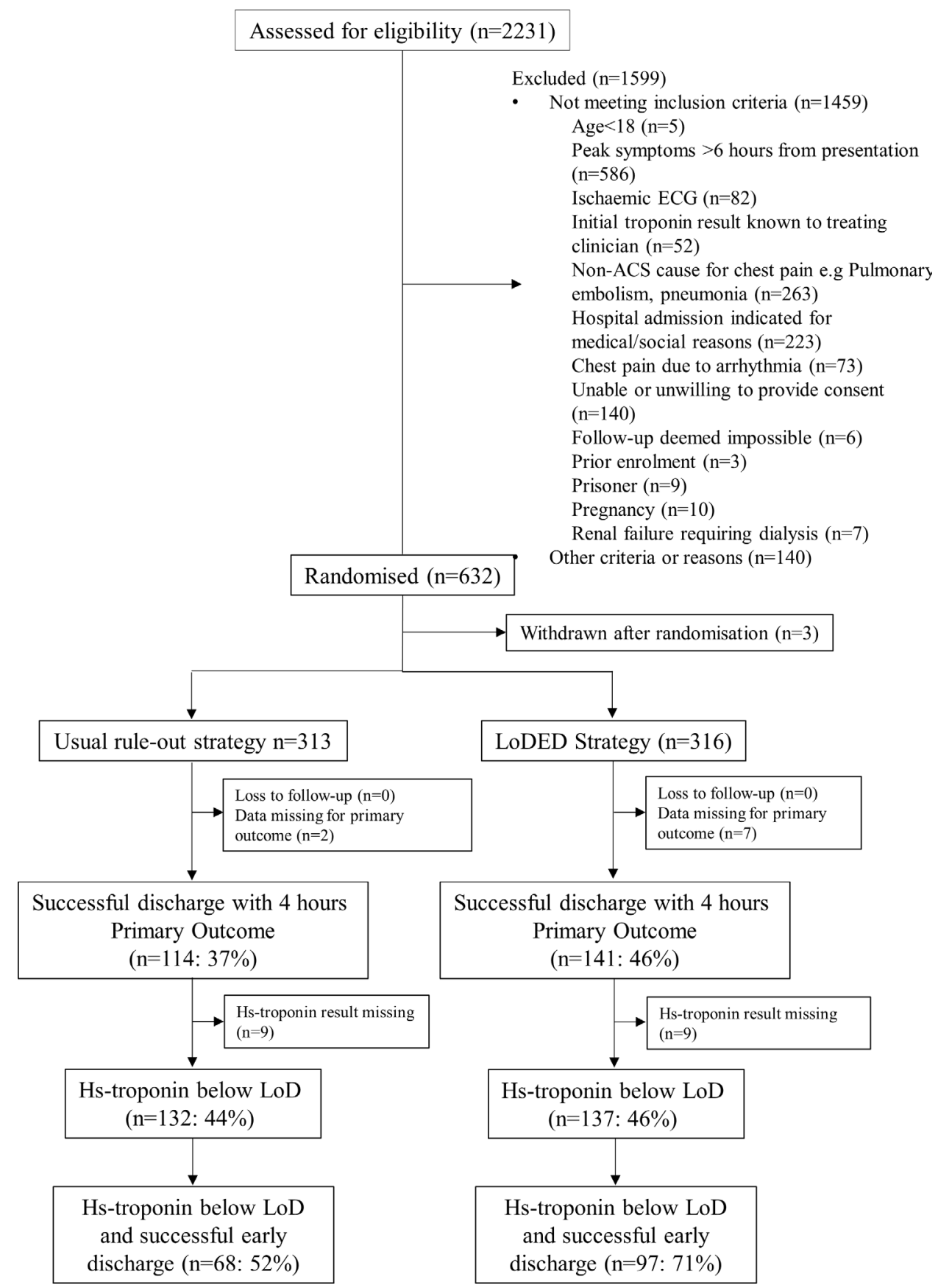

Figure 1 ConsolidatedStandards of Reporting Trials diagram. ACS, acute coronary syndrome; LoD, limit of detection; LoDED, limit of detection and ECG discharge.

\section{METHODS}

\section{Study design and participants}

The LoDED strategy versus usual care in adult patients with chest pain attending the ED trial was a prospective randomised open label blinded endpoint parallel group trial that aimed to assess the clinical effectiveness of the LoDED strategy in adult patients presenting to the ED with suspected cardiac chest pain in eight secondary and tertiary hospitals across England and Wales. Patients were eligible for recruitment if they were aged $\geq 18$ years; presented to the ED with chest pain and triggered a suspected cardiac chest pain investigation pathway (ie, the treating clinician intended to perform investigations to rule out an acute coronary syndrome); had a non-ischaemic ECG (no new T-wave inversion of $>3 \mathrm{~mm}$ or ST depression of
$>1 \mathrm{~mm}$ as judged by the treating clinician); and peak symptoms occurred $<6$ hours prior to ED presentation. Exclusion criteria are described in the published trial protocol and included in the Consolidated Standards of Reporting Trials (CONSORT) diagram (figure 1). ${ }^{20}$

\section{Randomisation and blinding}

Patients were randomised to evaluation with either the LoDED strategy (intervention) or the usual rule-out strategy in that site (control) in a 1:1 ratio, before initial hs-cTn results were known to the treating clinician. Randomisation was undertaken through a web-based portal, stratified by site and minimised by age and sex. 
Table 1 Summary of usual rule-out strategies (control) by site

\begin{tabular}{|c|c|c|c|c|}
\hline Site (All UK) & $\begin{array}{l}\text { High-sensitivity troponin } \\
\text { assay }\end{array}$ & $\begin{array}{l}\text { Risk stratification tool and } \\
\text { definition of low-risk }\end{array}$ & $\begin{array}{l}\text { Troponin assay sampling } \\
\text { times for low-risk patients }\end{array}$ & $\begin{array}{l}\text { Troponin assay result to allow discharge if } \\
\text { low-risk }\end{array}$ \\
\hline \multicolumn{5}{|c|}{ Sites not using the LoD cut-off within usual rule-out strategies } \\
\hline Royal United Hospital, Bath & Roche Elecsys hs-cTnT* & $\begin{array}{l}\text { GRACE }^{28} \text { score } \\
<1.5 \% \text { six month mortality }\end{array}$ & $\begin{array}{l}\text { Presentation and after } 6 \text { hours } \\
\text { (or } 6 \text { hours from onset of pain) }\end{array}$ & $\begin{array}{l}\text { Both results }<14 \mathrm{ng} / \mathrm{L} \text { OR } 14-30 \mathrm{ng} / \mathrm{L} \text { and no } \\
\text { change }>20 \% \text { between tests }\end{array}$ \\
\hline Southmead Hospital, Bristol & Roche Elecsys hs-cTnT* & TIMI score $<2$ & Presentation and after 2 hours & $\begin{array}{l}\text { Both results }<14 \mathrm{ng} / \mathrm{L} \text { OR } 14-30 \mathrm{ng} / \mathrm{L} \text { and no } \\
\text { change }>20 \% \text { between tests }\end{array}$ \\
\hline Derriford Hospital, Plymouth & Abbott Architect hs-cTnl† & HEART $^{26}$ score $\leq 3$ & Presentation and after 3 hours & Both results $<99$ th percentile (sex-specific) $\dagger$ \\
\hline $\begin{array}{l}\text { University Hospital, } \\
\text { Southampton }\end{array}$ & $\begin{array}{l}\text { Beckman-Coulter Access } \\
\text { hs-cTnl }\end{array}$ & $\mathrm{TIMI}^{29}$ score $<2$ & Presentation and after 3 hours & Both results $<18 \mathrm{ng} / \mathrm{L}$ \\
\hline \multicolumn{5}{|c|}{ Sites using the LoD cut-off within usual rule-out strategies } \\
\hline $\begin{array}{l}\text { University Hospital of Wales, } \\
\text { Cardiff }\end{array}$ & Abbott Architect hs-cTnl† & HEART $^{26}$ score $\leq 2$ & Presentation and after 3 hours & $\begin{array}{l}\text { Presentation }<2 \mathrm{ng} / \mathrm{L}(\mathrm{LOD}) \text { and }>2 \text { hours after } \\
\text { symptom onset } \\
\text { OR both results }<99 \text { th percentile (sex-specific) } \dagger\end{array}$ \\
\hline $\begin{array}{l}\text { Royal Devon and Exeter } \\
\text { Hospital, Exeter }\end{array}$ & Roche Elecsys hs-cTnT* & $\begin{array}{l}\text { Absence of ECG changes and } \\
\text { unstable angina symptoms }\end{array}$ & Presentation and after 1 hour & $\begin{array}{l}\text { Presentation }<5 \mathrm{ng} / \mathrm{L} \text { (LoD) and }>3 \text { hours after } \\
\text { symptom onset } \mathrm{OR} \text { both results }<14 \mathrm{ng} / \mathrm{L}\end{array}$ \\
\hline $\begin{array}{l}\text { Royal London Hospital, Barts } \\
\text { Health NHS Trust, London }\end{array}$ & Roche Elecsys hs-cTnT* & HEART $^{26}$ score $\leq 3$ & Presentation and after 3 hours & $\begin{array}{l}\text { Presentation }<5 \mathrm{ng} / \mathrm{L} \text { (LoD) OR both results } \\
<14 \mathrm{ng} / \mathrm{L}\end{array}$ \\
\hline $\begin{array}{l}\text { Royal Berkshire Hospital, } \\
\text { Reading }\end{array}$ & Roche Elecsys hs-cTnT* & HEART $^{26}$ score $\leq 3$ & Presentation and after 1 hour & $\begin{array}{l}\text { Presentation }<5 \mathrm{ng} / \mathrm{L} \text { (LoD) and }>3 \text { hours after } \\
\text { symptom onset } \mathrm{OR} \text { both results }<14 \mathrm{ng} / \mathrm{L}\end{array}$ \\
\hline
\end{tabular}

*Roche Elecsys hs-cTnT. This assay has a 99th percentile value of $14 \mathrm{ng} / \mathrm{L}$ and LoD of $5 \mathrm{ng} / \mathrm{L}$.

†Abbott Architect hs-cTnl. This assay has 99th percentile value of $34 \mathrm{ng} / \mathrm{L}$ in men and $16 \mathrm{ng} / \mathrm{L}$ in women and LoD of $2 \mathrm{ng} / \mathrm{L}$.

$\ddagger$ Beckman-Coulter Access hs-cTnl. This assay has a 99th percentile value of $18 \mathrm{ng} / \mathrm{L}$ and LoD of $2 \mathrm{ng} / \mathrm{L}$.

GRACE, Global Registry of Acute Coronary Events; HEART, history, ECG, age, risk factors, troponin; hs-cTnl, high-sensitivity cardiac troponin l; hs-cTnT, high-sensitivity cardiac troponin T; LOD, limit of detection; TIMI, Thrombolysis In Myocardial Infarction.

\section{Procedures}

\section{Cardiac troponin testing and measurement}

All participants had a blood sample taken for hs-cTn measurement at, or shortly after, ED arrival as part of their standard clinical assessment. Where repeat hs-cTn sampling was required, this was undertaken according to local guidelines (table 1) and not controlled for trial purposes.

Clinical blood samples were analysed, in real-time, locally in central hospital laboratories for either the Roche Elecsys highsensitivity cardiac troponin T (hs-cTnT) assay, Abbott Architect high-sensitivity cardiac troponin I (hs-cTnI) or Beckman Coulter Access hs-cTnI (further assay details in table 1).

\section{Trial interventions}

LoDED Strategy (intervention)

Participants randomised to the LoDED strategy were eligible for discharge if their ECG was non-ischaemic; a single hs-cTn test taken at presentation (and irrespective of the symptom onset time) was undetectable for the assay used at that study site; and there was no ongoing clinical concern. Any participant not fulfilling these discharge criteria reverted to the usual rule-out strategy at that study site.

\section{Usual rule-out strategies (control)}

Table 1 summarises the usual rule-out strategies in use across the eight sites. No sites used identical strategies; this heterogeneity accords with the findings of a cross-sectional survey of English hospitals and therefore reflects current practice. ${ }^{19}$

Clinical management, data collection and follow-up processes are detailed in the supplementary material and in the published protocol. ${ }^{20}$

\section{Outcomes}

The primary outcome was successful early discharge, defined as discharge from hospital within 4 hours of ED arrival, without a MACE occurring within 30 days. A time point was chosen for the primary outcome, rather than admission/discharge rate, because hospital admission is defined differently in different hospitals. This approach has been used in previous randomised trials evaluating the clinical effectiveness of rule-out strategies. $^{72}$ The time point of 4 hours was selected to reflect the NHS 4-hour ED standard. Such targets have been used internationally to provide an impetus to manage patients in a timely manner in an emergency setting. ${ }^{1}$ The safety endpoint of MACE occurring within 30 days was defined as cardiac death, type I AMI or emergency revascularisation occurring within 30 days of ED attendance (including the index presentation). Type I AMI was defined according to the Fourth Universal definition (a rise and/or fall of troponin above the 99th percentile). ${ }^{4}$ A significant rise and/or fall was defined as an absolute change in troponin over time of at least half the 99th percentile value of the assay in use. ${ }^{22}$ Primary outcomes were adjudicated by an independent expert panel comprising a senior emergency physician and cardiologist blinded to both participants' allocated groups and initial hs-cTn results. Secondary outcomes are listed in the supplement.

\section{Statistical analysis}

The statistical and health economic analysis are described in detail in the supplementary material. Briefly, for the statistical analysis of the primary outcome, an intention-to-diagnose logistic regression analysis, adjusting for age and sex using all those randomised with complete data, was conducted by centre. As expected, usual care pathways differed between sites, and the proportion of patients discharged within 4 hours varied, which influenced observed treatment effects at each centre. Meta-analysis methodology was used to produce an overall OR and between-centre heterogeneity examined using the $\mathrm{Q}$ and $\mathrm{I}^{2}$ statistic. Potential reasons for heterogeneity were investigated further for sites with and without the undetectable cut-off in usual care and by hs-cTn assay type. Kaplan-Meier-type graphs illustrate the relationship between length of hospital stay. 
Table 2 Patient demographics and risk characteristics

\begin{tabular}{|c|c|c|c|}
\hline & $\begin{array}{l}\text { Usual rule-out } \\
\text { strategy }(n=313)\end{array}$ & $\begin{array}{l}\text { LoDED strategy } \\
(\mathrm{n}=316)\end{array}$ & $\begin{array}{l}\text { All patients } \\
(\mathrm{n}=629)\end{array}$ \\
\hline Age (years), mean (SD) & $53.6(16.2)$ & $54.0(16.2)$ & $53.8(16.1)$ \\
\hline \multicolumn{4}{|l|}{ Sex } \\
\hline Women (\%) & $127(41)$ & $129(41)$ & $256(41)$ \\
\hline Men (\%) & $186(59)$ & $187(59)$ & $373(59)$ \\
\hline \multicolumn{4}{|l|}{ Ethnic origin $(\%)^{*}$} \\
\hline White & $277(89)$ & $277(88)$ & $554(88)$ \\
\hline \multicolumn{4}{|c|}{ Chest pain history (clinician reported) $†(\%)$} \\
\hline Slightly suspicious & $176(56)$ & $171(54)$ & $347(55)$ \\
\hline Moderately suspicious & $112(36)$ & $111(35)$ & $223(36)$ \\
\hline Highly suspicious & $25(8)$ & $34(11)$ & $59(9)$ \\
\hline $\begin{array}{l}\text { Prior history of coronary artery } \\
\text { disease (\%) }\end{array}$ & $41(13)$ & $40(13)$ & $81(13)$ \\
\hline \multicolumn{4}{|l|}{ Known risk factors (\%) } \\
\hline Hypercholesterolaemia & $48(15)$ & $55(17)$ & $103(16)$ \\
\hline Hypertension & $86(28)$ & $82(26)$ & $168(27)$ \\
\hline Diabetes (treated) & $31(10)$ & $34(11)$ & $65(10)$ \\
\hline Current smoking & $54(17)$ & $54(17)$ & $108(17)$ \\
\hline $\begin{array}{l}\text { Family history of coronary artery } \\
\text { disease (first-degree relative } \\
\text { under the age of } 65 \text { years) }\end{array}$ & $67(21)$ & $71(23)$ & $138(22)$ \\
\hline TIMI score, $\neq$ median (IQR) & $0(0-1)$ & $0(0-1)$ & $0(0-1)$ \\
\hline TIMI score $0(\%)$ & $175(57.6)$ & $176(57.3)$ & $351(57.5)$ \\
\hline TIMI score $1(\%)$ & $60(19.7)$ & $63(20.5)$ & $123(20.1)$ \\
\hline TIMI score $\geq 2(\%)$ & $69(22.7)$ & $68(22.2)$ & $137(22.4)$ \\
\hline HEART score, $\S$ median (IQR) & $2(1-4)$ & $2(1-4)$ & $2(1-4)$ \\
\hline HEART score 0-3 (\%) & $213(71)$ & $211(69)$ & $424(70)$ \\
\hline HEART score 4-6 (\%) & $75(25)$ & $83(27)$ & $128(26)$ \\
\hline HEART score $\geq 7(\%)$ & $13(4)$ & $11(4)$ & $24(4)$ \\
\hline $\begin{array}{l}\text { Chest pain onset to arrival in ED } \\
\text { (hours), median (IQR) }\end{array}$ & $2.5(1.6-3.9)$ & $2.2(1.4-3.4)$ & $2.3(1.5-3.6)$ \\
\hline $\begin{array}{l}\text { Chest pain onset to arrival in ED } \\
\text { (hours) (range) } q\end{array}$ & $0.02-178.4$ & $0.02-120.5$ & $0.02-178.4$ \\
\hline $\begin{array}{l}\text { Chest pain onset to first hs-cTn } \\
\text { sample collection (hours) (median } \\
\text { (IQR)) }\end{array}$ & $3.3(2.4-4.7)$ & $3.2(2.2-4.5)$ & $3.3(2.3-4.5)$ \\
\hline $\begin{array}{l}\text { Chest pain onset to first hs-cTn } \\
\text { sample collection (hours) (range) }\end{array}$ & $0.2-178.6$ & $0.5-121.4$ & $0.2-178.6$ \\
\hline \multicolumn{4}{|c|}{ Chest pain onset to first hs-cTn sample collection categorised by minutes (\%) } \\
\hline $0-60 \mathrm{~min}$ & $9(3)$ & $4(1)$ & $13(2)$ \\
\hline $61-120 \min$ & $34(12)$ & $54(18)$ & $88(15)$ \\
\hline $121-180 \mathrm{~min}$ & $85(29)$ & $76(26)$ & $161(28)$ \\
\hline$>181 \mathrm{~min}$ & $161(56)$ & $162(55)$ & $323(55)$ \\
\hline
\end{tabular}

* Data missing for one patient.

tClinicians were asked to grade level of suspicion after taking a history according to definitions used within the HEART score (see further). ${ }^{26} \mathrm{~A}$ highly suspicious history included all of central chest discomfort with radiation to the jaw/arms precipitated by exertion and relieved by rest or nitrates. A moderately suspicious history included some highly suspicious features but will have some other atypical features, such as right-sided pain, burning pain or pain that is worse on deep inspiration. A slightly suspicious history did not have any highly suspicious features but by definition of being involved in the trial, the treating clinician felt the patient warranted testing to rule out a cardiac cause for chest pain.

\$TIMI score. ${ }^{29}$ Data available in $611 / 629$ cases.

§HEART: data available in $606 / 629$ cases $^{26}$

IRefers to chest pain onset time, rather than peak symptoms. Note patients were excluded if peak symptoms occurred $>6$ hours prior to ED presentation.

ED, emergency department; HEART, History, ECG, Age, Risk factors, Troponin; hs-cTn, highsensitivity cardiac troponin; LoDED, limit of detection and ECG discharge; TIMI, Thrombolysis In Myocardial Infarction.

\section{Sample size}

The target sample size was based on both observational and interventional data. ${ }^{73-15}$ For the overall population, we anticipated $8 \%$ of participants would be discharged within 4 hours using usual care and at least $17 \%$ using the LoDED strategy. Therefore, the trial was powered to detect a $9 \%$ difference between early discharge rates with $90 \%$ power and two-sided alpha of 0.05 . This required 282 patients in each group with primary outcome data. Allowing for a $95 \%$ follow-up rate, a total recruitment target of 594 participants was set.

\section{Patient and public involvement}

Patient and public involvement occurred at all stages of trial design and conduct. A patient advisory group (PAG) oversaw development and approval of patient-facing materials. The PAG supported the interpretation of results and approved the final manuscript submission.

\section{RESULTS}

In total, 632 patients were randomised between 4 June 2018 and 4 March 2019. Three patients were later withdrawn; one had no record of consent and two were randomised in error. All remaining 629 patients were successfully monitored for 30 days. A total of 316 patients were allocated to the LoDED strategy and 313 to usual care (figure 1). The baseline characteristics were well balanced between groups (table 2). The cohort was predominantly men (59\%), of white British ethnicity (88\%), with a mean age of 53.8 years, and were at low risk of cardiac disease. Patients presented at a median of 2.3 hours (IQR 1.5-3.6 hours) after chest pain onset and the first hs-cTn sample was drawn at a median of 3.3 hours (IQR 2.3-4.5) after chest pain onset. Of the 629 patients, $42(7 \%)$ had a MACE within 30 days (table 3).

\section{Primary outcome}

Data were available for the primary outcome in $620 / 629$ patients. Discharge time was not recorded for 14 patients, but for five of these, their length of stay was over 4 hours. When allocated to the LoDED strategy, 141 of 309 (46\%) were discharged within 4 hours of arrival, without subsequent 30-day MACE, and for usual rule-out strategies, 114 of 311 (37\%) (table 3); pooled OR 1.58 (95\% CI 0.84 to 2.98). The Kaplan-Meier-type length of stay curve illustrates improved discharge rates using the LoDED strategy, which persisted until 8 hours after patient arrival (figure 2).

Figure 3 demonstrates significant heterogeneity between sites for the primary outcome $\left(\mathrm{I}^{2}=61 \%, \mathrm{p}=0.01\right.$ for $\mathrm{Q}$ test of heterogeneity), which was only partially explained by the control rule-out strategies in use. For sites using the undetectable cut-off within usual care, the pooled OR was 0.93 (95\% CI 0.54 to $1.59, \mathrm{I}^{2}=0.0 \%, \mathrm{p}=0.58$ for $\mathrm{Q}$ test). For sites not using the undetectable cut-off within usual care, the pooled OR for the primary outcome was $2.87\left(95 \%\right.$ CI 1.07 to $7.69, \mathrm{I}^{2}=67 \%, \mathrm{p}=0.03$ for $\mathrm{Q}$ test). This positive treatment effect was driven by only two sites, Bristol (OR 8.33, 95\% CI 2.82 to 24.64) and Southampton (OR 6.01, 95\% CI 1.19 to 30.44), with no effect seen in the remaining two sites not using the LoD within usual care, Bath (OR 1.59, 95\% CI 0.60 to 4.25) and Plymouth (OR 1.14, 95\% CI 0.42 to 3.06). Kaplan-Meier-type length of stay curves for each site are included in online supplementary figures 1-8. Clinical outcomes by group allocation and site are shown in table 3 . A subgroup analysis of patients with an initial undetectable hs-cTn is detailed in figure 4 and in the online supplementary material. Resource use data are described in table 4, together with mean per-patient costs by category. Comparative costs are described in detail in the online supplementary material.

\section{Acceptability and adherence to the LoDED strategy}

Interviews with 11 patients from four sites and two focus groups with $20 \mathrm{ED}$ professionals and general practitioners were 
Table 3 Clinical outcomes and comparative costs

\begin{tabular}{|c|c|c|c|}
\hline & Usual rule-out strategy & LoDED strategy & \\
\hline \multicolumn{4}{|l|}{ All patients } \\
\hline Patients successfully discharged within 4 hours ( $1^{\circ}$ outcome) $(\%)$ & $114 / 311(37)$ & $141 / 309(46)$ & OR $1.58(95 \% \mathrm{Cl} 0.84 \text { to } 2.98)^{*}$ \\
\hline MACEs at 30 days (\%) & $17 / 313(5)$ & $25 / 316(8)$ & OR $1.50(95 \%$ Cl 0.76 to 3.02$) \dagger$ \\
\hline MACEs in patients discharged within 4 hours (\%) & $1 / 115(1)$ & $0 / 141(0)$ & - \\
\hline Median time to discharge (hours) (IQR) & $5.0(3.4-7.4)$ & $4.4(3.2-6.8)$ & All patients: $4.8(3.3-7.0)$ \\
\hline Patient satisfaction score, median (IQR)‡ & $50(44-55)$ & $51(44-55)$ & - \\
\hline \multirow[t]{2}{*}{ Mean secondary care costs up to 30 days post discharge $(95 \% \mathrm{Cl}) \S$} & $\mathrm{f527}(\mathrm{f} 391$ to $f 720)$ ๆ & $f 429$ ( $\mathrm{f} 302$ to $\mathrm{f} 651)$ ๆ & $\begin{array}{l}\text { Unadjusted incremental cost } \\
\mathrm{f} 98(\mathrm{f}-131 \text { to } \mathrm{f} 332)^{* *}\end{array}$ \\
\hline & & & $\begin{array}{l}\text { Adjusted incremental cost: } \\
\mathrm{f} 151(\mathrm{f}-34 \text { to } \mathrm{f} 336)+\dagger\end{array}$ \\
\hline \multicolumn{4}{|l|}{ Patients with an initial hs-cTn below the LoD $\neq \ddagger$} \\
\hline Patients with an initial hs-cTn below the LoD (\%) & 133/304 (44) & $141 / 307(46)$ & All patients: $274 / 611(45)$ \\
\hline Patients with an initial hs-cTn below the LoD successfully discharged within 4 hours (\%) & $68 / 132(52)$ & $97 / 137(71)$ & OR $2.51(95 \% \mathrm{Cl} 0.76$ to 8.25$) \dagger$ \\
\hline MACEs at 30 days & 0 & 0 & - \\
\hline Median time to discharge (hours) (IQR) & $4.0(3.0-5.7)$ & $3.5(2.7-4.3)$ & All patients: $3.7(2.8-5.2)$ \\
\hline Mean primary and secondary care costs up to 30 days post discharge $(95 \% \mathrm{Cl}) \S \S$ & 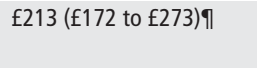 & $\mathrm{f} 184$ (f153 to 243)ף & $\begin{array}{l}\text { Incremental cost: } \\
\mathrm{f} 29(-\mathrm{f} 33 \text { to } f 99)^{* *}\end{array}$ \\
\hline \multicolumn{4}{|l|}{ Site variation } \\
\hline \multicolumn{4}{|l|}{ Patients successfully discharged within 4 hours $\left(1^{\circ}\right.$ outcome) $(\%)$} \\
\hline Royal United Hospital, Bath & $16 / 34(47)$ & $20 / 35(57)$ & \\
\hline Southmead Hospital, Bristol & $6 / 60(10)$ & $25 / 60(42)$ & \\
\hline Derriford Hospital, Plymouth & $10 / 46(22)$ & $11 / 45(24)$ & \\
\hline University Hospital, Southampton & $3 / 27(11)$ & 9/26 (35) & \\
\hline University Hospital of Wales, Cardiff & $11 / 17(65)$ & $11 / 21(52)$ & \\
\hline Royal Devon and Exeter Hospital, Exeter & $21 / 49(43)$ & $20 / 48(42)$ & \\
\hline Royal London Hospital, Barts Health NHS Trust, London & $8 / 34(24)$ & $11 / 33(33)$ & \\
\hline Royal Berkshire Hospital, Reading & $39 / 44(89)$ & $34 / 41(83)$ & \\
\hline \multicolumn{4}{|l|}{ Median time to discharge (hours) (IQR) } \\
\hline Royal united Hospital, Bath & 4.4 (3.1 to 9.7$)$ & $3.5(2.5$ to 6.6$)$ & \\
\hline Southmead Hospital, Bristol & $5.6(4.7$ to 7.1$)$ & $4.5(3.2$ to 6.1$)$ & \\
\hline Derriford Hospital, Plymouth & $6.1(4.3$ to 7.0$)$ & $5.5(4.1$ to 7.1$)$ & \\
\hline University Hospital, Southampton & $5.9(4.9$ to 8.3$)$ & $5.7(3.4$ to 6.2$)$ & \\
\hline University Hospital of Wales, Cardiff & $3.8(2.8$ to 6.5$)$ & $4.0(3.1$ to 5.8$)$ & \\
\hline Royal Devon and Exeter Hospital, Exeter & 5.1 (3.5 to 26.5$)$ & 5.3 (3.5 to 28.4$)$ & \\
\hline Royal London Hospital, Barts Health NHS Trust, London & $5.4(4.1$ to 9.1$)$ & $6.4(3.9$ to 8.2$)$ & \\
\hline Royal Berkshire Hospital, Reading & $2.8(2.5$ to 3.4$)$ & $3.2(2.5$ to 3.8$)$ & \\
\hline
\end{tabular}

*Pooled adjusted OR.

†Pooled unadjusted OR.

¥The Group Health Association of America Satisfaction Questionnaire was completed on discharge (online supplementary table S4). ${ }^{27}$ Scores out of 5 were summed for each of 11 questions, giving a maximum score of 55. 352/629 responses received.

§Data were available to calculate secondary care costs in 295/313 patients in the usual care arm and 295/316 in the intervention. The unit cost template for this analysis is available in the online supplementary material.

IMean cost with $95 \%$ bias corrected and accelerated $\mathrm{Cls}$.

**Bootstrap mean difference with bias $95 \%$ bias corrected and accelerated Cls.

t†Adjusted predicted mean cost difference (95\% Confidence Intervals), generalised linear model (GLM) with a log link function and inverse gaussian family.

$\ddagger \ddagger D$ ata missing for 18 patients on the initial troponin result: 15 samples were haemolysed and three were lost.

$\S \S$ Data were available to calculate primary and secondary care costs in 125/133 patients in the usual care arm 122/141 in the intervention. The unit cost template for this analysis is available in the online supplementary material.

Hs-cTn, high-sensitivity cardiac troponin; LoD, limit of detection; LoDED, limit of detection and ECG discharge; MACE, major adverse cardiac event.

conducted. From the qualitative interviews, patients were not anxious about the new strategy, trusted the clinicians' judgement and welcomed the opportunity to be discharged sooner. Clinicians were more sceptical about discharging patients early in case they missed something and awaited the trial results to reassure them that there were no adverse consequences of discharging patients sooner (a full qualitative report has been submitted for publication and is available on request to the authors). Despite this finding, $88 \%$ (95\%CI $81 \%$ to $93 \%)$ of patients allocated to the LoDED strategy who had an initial hs-cTn below the LoD were discharged from hospital in accordance with the strategy.

\section{DISCUSSION}

We evaluated whether the use of a rapid rule-out strategy based on a single undetectable hs-cTn test taken on arrival at the ED (irrespective of chest pain onset time), together with a normal ECG (the LoDED strategy), increased the rate of safe, early discharge. When embedded within existing rule-out strategies in use across eight sites, the LoDED strategy facilitated discharge within 4 hours in over $40 \%$ of all patients and, importantly, no patient discharged with an initial undetectable hs-cTn had a MACE before 30 days. This accords with findings from multiple observational studies that the LoDED strategy is safe and provides clinicians with an opportunity to discharge 


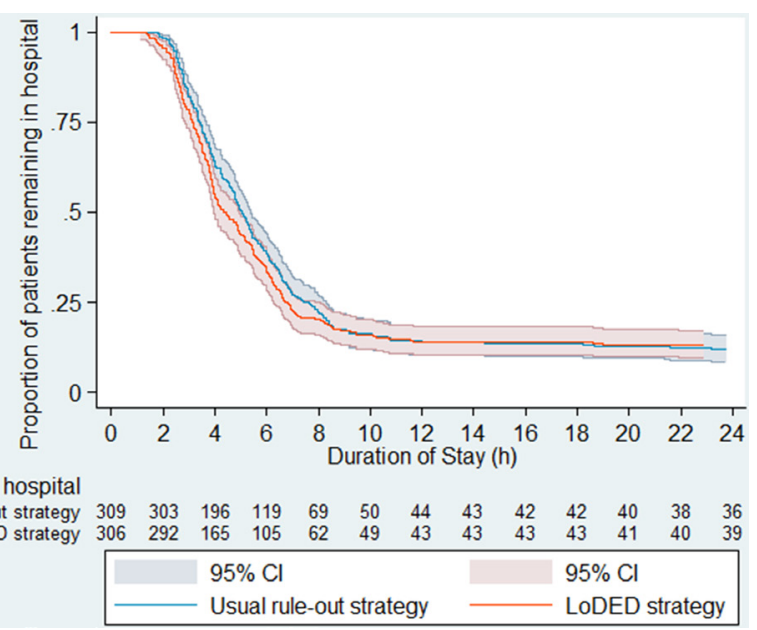

Figure 2 Kaplan-Meier-type length of stay curve by group allocation (intention to treat). LoDED, limit of detection and ECG discharge.

low-risk patients based on a single undetectable hs-cTn result. ${ }^{8-15}$ However, when compared with existing rule-out strategies which use hs-cTn assays as recommended by consensus guidelines, ${ }^{19} 20$ the LoDED strategy did not show a difference in the rate of safe early discharge, even in those patients with an initial undetectable hs-cTn. There was also no difference in healthcare costs between the two strategies.

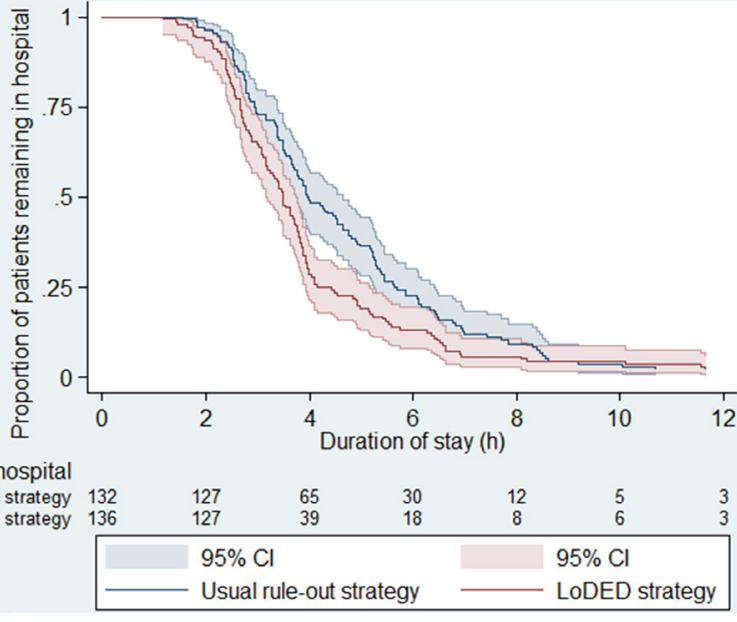

Figure 4 Kaplan-Meier-type length of stay curve for patients with an initial high-sensitivity cardiac troponin below the limit of detection. LoDED, limit of detection and ECG discharge.

There are a number of potential explanations for these findings. First, the proportion of patients discharged within 4 hours across control strategies (37\%) was much greater than the $8 \%$ estimated in our power calculation, which was based on an interventional study of a testing strategy of 0 and 2 hours undertaken 5 years prior to designing this trial (the primary

Study

Odds

ratio $(95 \% \mathrm{Cl})$
Weight

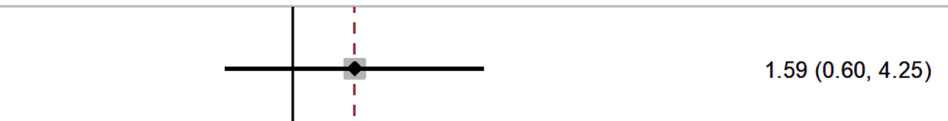

Bristol

Plymouth

Southampton

Cardiff

Exeter

Barts

Reading

Overall (I-squared $=60.5 \%, p=0.013$ )

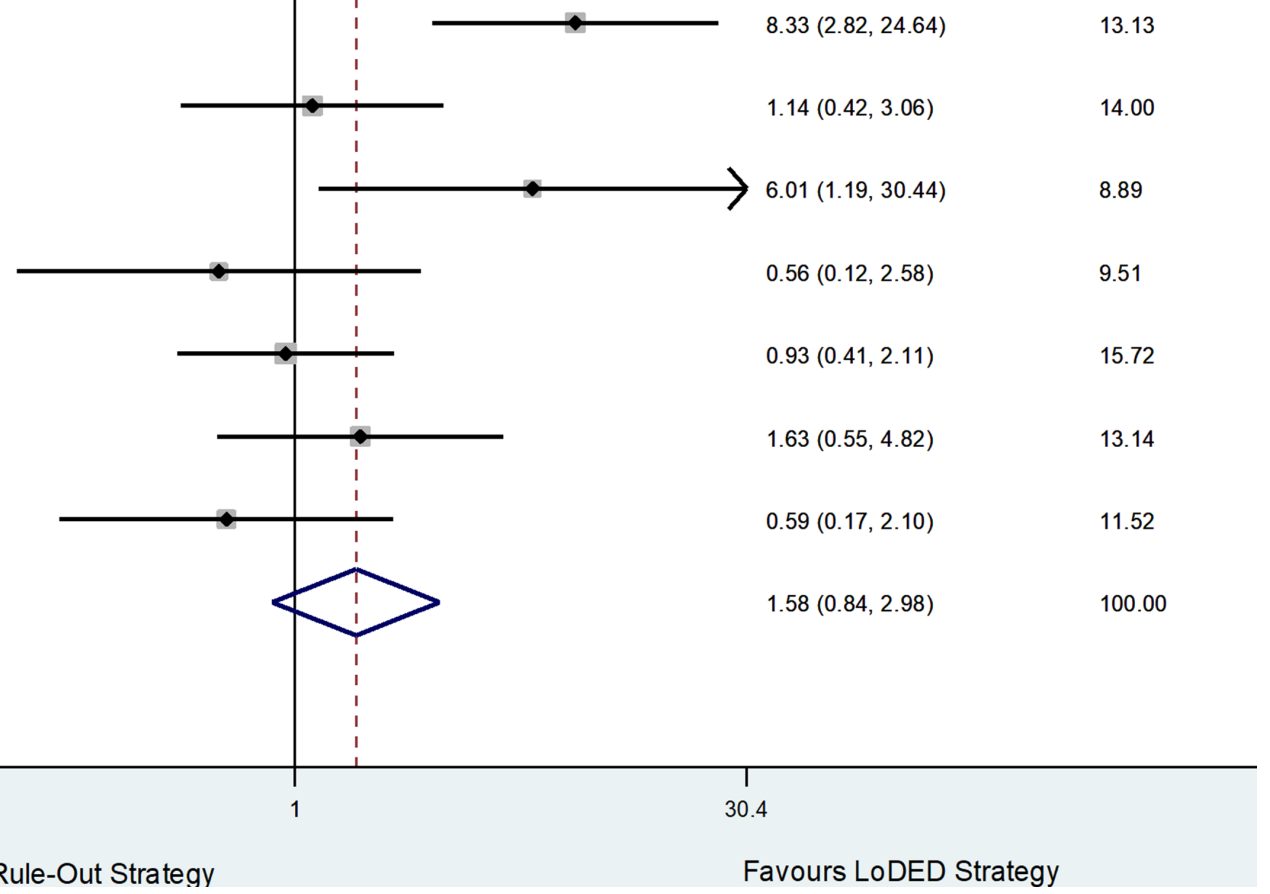

NOTE: Weights are from random effects analysis

$\frac{1}{.0329}$

Figure 3 Forest plot for the primary outcome of successful early discharge. LoDED, limit of detection and ECG discharge. 
Table 4 Total resource use and mean per-patient costs by category

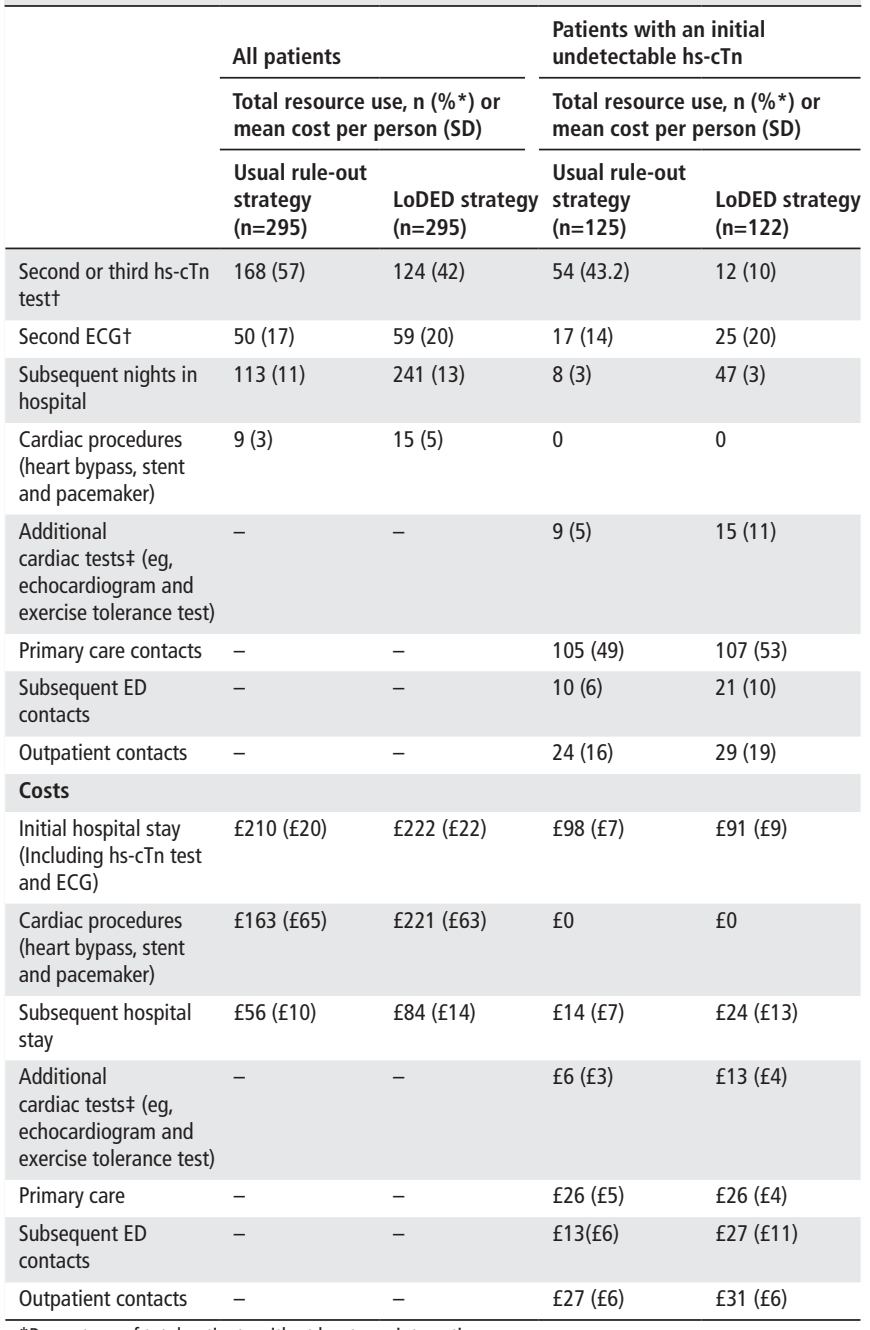

*Percentage of total patients with at least one interaction or resource use. $\ddagger$ Additional tests were (\% undergoing test in the whole cohort) echocardiogram (4.9\%), exercise tolerance test $(0.8 \%)$, stress echocardiogram $(0.4 \%)$, CT coronary angiogram $(0.8 \%)$, invasive coronary angiogram $(0.8 \%)$, 24-hour cardiac monitor $(0.8 \%)$ and abdominal ultrasound $(0.4 \%)$.

ED, emergency department; hs-cTn, high-sensitivity cardiac troponin; LoDED, limit of detection and ECG discharge.

outcome for that trial was successful discharge within 6-hour of arrival; data on 4-hour discharge rates were provided through personal communication with the trial statistician). ${ }^{7}$ This may reflect an improved clinical understanding of the use of rapid rule-out strategies to facilitate early discharge and subsequent guideline recommendations that the undetectable cut-off can be used in clinical practice, although with caveats. ${ }^{17} 18$ Contamination, whereby clinicians simply applied the LoDED strategy to those patients allocated to usual care, is also possible. Second, we observed considerable heterogeneity between sites, with some sites demonstrating a potential for reduced effectiveness of the intervention strategy compared with usual care. We demonstrated improvements in the primary outcome at some sites where the undetectable cut-off was not already in use, as per guideline recommendations. However, even when accounting for these control strategy differences, significant heterogeneity remained. This is an important finding and may reflect local culture, in terms of which patients are selected for rule-out testing, and the high clinical intensity of the ED, which are beyond the rule-out strategy itself. Third, we undertook this study, in which discharge within 4 hours was a component of the primary outcome, during a time period when performance against the 4-hour standard for all ED patients has been at its worst for a decade. ${ }^{1}$ Given that we observed separation on the Kaplan-Meier-type length of stay curves between usual care and the LoDED strategy up to 8 hours after ED attendance, our findings may reflect poor performance against this standard, rather than performance of the rule-out strategy itself.

Despite being available for nearly a decade, there are very few interventional trials evaluating the clinical effectiveness or cost-effectiveness of hs-cTn assays in the rapid discharge of patients with chest pain admitted to the ED. One randomised controlled trial of over 3000 participants evaluating the ESCrecommended Roche hs-cTnT assay of 0 and 1 hour, rule-out strategy demonstrated improved clinical effectiveness in terms of rapid discharge from the ED, but compared this strategy to a control group where contemporary cut-offs were used, which may have inflated the results. ${ }^{23}$

Our trial has several strengths that distinguish it from prior studies and extend the generalisability of the findings. First, we tested a strategy that does not rely on a risk score. Numerous risk scores such as thrombolysis in myocardial infarction, history, ECG, age, risk factors, troponin and troponin-only Manchester acute coronary syndromes have been evaluated and shown promise in observational studies. ${ }^{24} 25$ However, when tested within interventional trials, poor adherence by clinicians has limited their clinical effectiveness when applied to practice. ${ }^{726}$ Our results demonstrate good adherence to the LoDED strategy, suggesting clinicians would be willing to follow its recommendations when used in routine care. Second, one of the remaining controversies in the use of the undetectable cut-off is in patients who present early after chest pain onset. ${ }^{14}$ By excluding patients who presented over 6 hours from chest pain onset, we have tested the LoDED strategy in a population where around $45 \%$ of patients had their first sample drawn within 3 hours of chest pain onset. Given there were no 30-day MACEs in patients with an initial undetectable troponin in either arm of the trial, this provides reassurance to clinicians that the LoDED strategy can be used irrespective of chest pain onset time. However, clinical judgement should be used to ascertain the need for serial testing in very early presenters. Third, previous trials have tested hs-cTn assays provided by a single manufacturer. ${ }^{23}$ This approach can have significant limitations in terms of generalisability because hospitals may have limited opportunity to change assays. By applying the LoDED strategy across the three different troponin assays in which the undetectable cut-off has been evaluated in observational studies, we have enhanced the generalisability of our findings. ${ }^{8-15}$

Other study limitations include a trial design that assessed the clinical effectiveness of the LoDED strategy, rather than comparing safety between the intervention and control arms. It is possible that some patients discharged according to the LoDED strategy may have developed a subsequent subclinical rise in troponin after discharge. It is known that hs-cTn elevations above the undetectable cut-off may have prognostic implications when follow-up periods are extended beyond a year. ${ }^{27}$ Prognostic implications have not been addressed in our trial. Finally, despite randomising participants prior to hs-cTn results being known, the population demographics suggest this is a lowrisk chest pain population. We excluded patients with ischaemic ECG findings, which may explain the low-risk demographics. However, the trial sample had lower-risk demographics than an unselected observational study, which applied the same ECG exclusion criteria. ${ }^{8}$ Therefore, it is possible that a degree of patient selection occurred, and the study findings can only be 


\section{Key messages}

\section{What is already known on this subject?}

- Numerous cohort studies, systematic reviews and metaanalyses suggest that patients with an undetectable high-sensitivity cardiac troponin (hs-cTn) concentration at presentation to the emergency department (ED) are at lowrisk of 30-day major adverse cardiac events. This finding has been repeated across different troponin assays. However, a lack of interventional trial data means that the clinical effectiveness of embedding this rule-out strategy within routine practice is unknown.

\section{What might this study add?}

- Although the limit of detection (LoD) strategy facilitated the safe early discharge of over $40 \%$ of low-risk patients with chest pain, clinical effectiveness was not statistically different from usual care strategies that already incorporate highsensitivity troponin tests. This finding brings into question existing observational evidence and may, in part, be due to factors beyond the strategy itself, such as the high clinical intensity of the ED, which has an important effect on the ability of clinicians to facilitate early discharge.

\section{How might this impact on clinical practice?}

- The LoD strategy is safe and provides clinicians with an opportunity to discharge low-risk patients based on a single undetectable hs-cTn result taken at presentation to the ED. However, clinicians should be mindful of the importance of clinical judgement in patients presenting early after chest pain onset, and in some centres the strategy may not lead to improved clinical effectiveness. Long-term prognostic implications of this strategy have not been addressed in this trial.

applied to patients with chest pain deemed low risk by treating clinicians.

We have shown that a rule-out strategy based on an initial undetectable hs-cTn test, normal ECG and no ongoing clinical concern (LoDED), facilitates the safe early discharge of over $40 \%$ of patients with chest pain, without an increase in healthcare costs. However, the clinical effectiveness of such a strategy is limited when compared with existing rule-out strategies, especially those that already incorporate the undetectable cut-off. In addition, the clinical effectiveness of early rule-out strategies is likely to be undermined by a range of system factors such as prolonged ED waiting times and crowding.

\footnotetext{
Author affiliations

Emergency Department, North Bristol NHS Trust, Bristol, UK

${ }_{2}^{2}$ Population Health Sciences, Bristol Medical School, University of Bristol, Bristol, UK ${ }^{3}$ Research Design Service South West, University Hospitals Bristol NHS Foundation Trust, Bristol, UK

${ }^{4}$ Peninsula Clinical Trials Unit, University of Plymouth, Plymouth, UK

${ }^{5}$ Cardiology, North Bristol NHS Trust, Westbury on Trym, UK

${ }^{6}$ Biochemistry, North Bristol NHS Trust, Westbury on Trym, UK

${ }^{7}$ Emergency Department, Royal Devon and Exeter NHS Foundation Trust, Exeter, UK

${ }^{8}$ Emergency Department, Derriford Hospital, Plymouth, UK

${ }^{9}$ Academic Department of Military Emergency Medicine, Royal Centre for Defence

Medicine (Research and Academia), Birmingham, UK

${ }^{10}$ Centre for Medical Statistics, Plymouth University, Plymouth, UK

${ }^{1}$ Academic Department of Emergency care, The University Hospitals NHS Foundation trust, Bristol, UK

${ }^{12}$ Faculty of Health and Life Sciences, The University of the West of England, Bristol, UK
}

Correction notice Since the online publication of this article, the provenance and peer review statement has been updated to show that this article was externally peer reviewed.

\section{Twitter Edward Watts Carlton @eddcarlton}

Acknowledgements The British Heart Foundation assisted in providing an independent patient and public representative for the Trial Steering Committee. The Peninsula Clinical Trials Unit at the University of Plymouth provided support with the design, management and logistics of the trial. We thank the following individuals for their support during the conduct of the trial: Gareth Williams (Swansea), patient and public involvement lead; Jamie Milton, Gay and Adrian Sawyer (Bristol), members of the patients advisory group; Saif Al-Nahas, local principal investigator, Royal Unit Hospital, Bath, UK; Tim Rainer and Nick Manville, local principal investigators, University Hospital of Wales, Cardiff, UK; Manish Thakker, local principal investigator, Royal Berkshire Hospital, Reading, UK; Sanjay Ramamoorthy, local principal investigator, University Hospital Southampton, UK; Ben Bloom, Royal London Hospital, Barts Health NHS Trust, London; Research nursing staff at all sites, who ensured recruitment to time and target; Richard Body, independent emergency physician and chair of the Trial Steering Committee (TSC); James Gamble, independent cardiologist on the TSC; Sean Ewings, independent statistician on the TSC; Sarah Miles, patient representative on the TSC; Eva Sammut, cardiologist, Outcome Adjudication Committee; Anne Frampton, emergency physician, Outcome Adjudication Committee.

Contributors EWC conceived the idea for the study. EWC, JK, AR, JES and JRB were responsible for the initial study design, which was refined with the help of RK, JI and HT and support from our regional National Institute for Health Research Research Design Service. Expert advice on cardiological and biochemical elements of the study design was provided by SA and PB, respectively. HT provided the statistical plan and undertook data analysis. RK led the economic study and provided oversight to JG, who undertook the resource and cost analyses. JI developed the integrated qualitative study, with support from $\mathrm{LB}$, and supported the public and patient views in the study development. SCa and RC coordinated the study set-up and finalisation of the study protocol as senior trial manager and assistant trial manager, with oversight from SCr, director of the Peninsula Clinical Trials Unit. All authors contributed to the final study design and protocol development, critically revised successive drafts of the manuscript and approved the final version. The tria management group was responsible for the conduct of the study, overseen by the Trial Steering Committee.

Funding This trial was funded by the National Institute for Health Research (NIHR) through its Research for Patient Benefit programme (grant reference number PBPG-0416-20012). JRB is an NIHR senior investigator.

Disclaimer The views expressed are those of the author(s) and not necessarily those of the NIHR or the Department of Health and Social Care.

Competing interests $E C$ has received funding from Abbott Diagnostics for related research and honoraria for education from Roche.

Patient and public involvement Patients and/or the public were involved in the design, conduct, reporting or dissemination plans of this research. Refer to the Methods section for further details.

\section{Patient consent for publication Not required.}

Ethics approval The study was approved by the South West Frenchay Research Ethics Committee (REC reference 18/SW/0038) and each of the participating NHS Trust's Research and Development department. All patients provided written informed consent.

Provenance and peer review Not commissioned; externally peer reviewed.

Data availability statement Data are available upon reasonable request. Summary data collected from the study, including de-identified patient level data, can be made available upon request to the corresponding author.

Open access This is an open access article distributed in accordance with the Creative Commons Attribution Non Commercial (CC BY-NC 4.0) license, which permits others to distribute, remix, adapt, build upon this work non-commercially, and license their derivative works on different terms, provided the original work is properly cited, appropriate credit is given, any changes made indicated, and the use is non-commercial. See: http://creativecommons.org/licenses/by-nc/4.0/.

\section{ORCID iD}

Edward Watts Carlton http://orcid.org/0000-0002-2064-4618

\section{REFERENCES}

1 The Health and Social Care Information Centre. Hospital Accident \& Emergency Activity 2018-19, 2019. Available: http://www. hesonline. nhs. uk 
2 Goodacre S, Cross E, Arnold J, et al. The health care burden of acute chest pain. Heart 2005:91:229-30.

3 Thokala P, Goodacre SW, Collinson PO, et al. Cost-effectiveness of presentation versus delayed troponin testing for acute myocardial infarction. Heart 2012;98:1498-503.

4 Thygesen K, Alpert JS, Jaffe AS, et al. Fourth universal definition of myocardial infarction (2018). J Am Coll Cardiol 2018;72:2231-64.

5 Apple FS, Collinson PO, IFCC Task Force on Clinical Applications of Cardiac Biomarkers. Analytical characteristics of high-sensitivity cardiac troponin assays. Clin Chem 2012;58:54-61.

6 Reichlin T, Hochholzer W, Bassetti S, et al. Early diagnosis of myocardial infarction with sensitive cardiac troponin assays. N Eng/ J Med 2009;361:858-67.

7 Than MP, Pickering JW, Aldous SJ, et al. Effectiveness of EDACS versus ADAPT accelerated diagnostic pathways for chest pain: a pragmatic randomized controlled trial embedded within practice. Ann Emerg Med 2016;68:93-102.

8 Carlton EW, Cullen L, Than M, et al. A novel diagnostic protocol to identify patients suitable for discharge after a single high-sensitivity troponin. Heart 2015;101:1041-6.

9 Body R, Carley S, McDowell G, et al. Rapid exclusion of acute myocardial infarction in patients with undetectable troponin using a high-sensitivity assay. J Am Coll Cardiol 2011:58:1332-9.

10 Bandstein N, Ljung R, Johansson M, et al. Undetectable high-sensitivity cardiac troponin T level in the emergency department and risk of myocardial infarction. J Am Coll Cardiol 2014;63:2569-78.

11 Zhelev Z, Hyde C, Youngman E, et al. Diagnostic accuracy of single baseline measurement of Elecsys troponin T high-sensitive assay for diagnosis of acute myocardial infarction in emergency department: systematic review and meta-analysis. BMJ 2015;350:h15.

12 Body R, Burrows G, Carley S, et al. High-sensitivity cardiac troponin T concentrations below the limit of detection to exclude acute myocardial infarction: a prospective evaluation. Clin Chem 2015:61:983-9.

13 Carlton E, Greenslade J, Cullen L, et al. Evaluation of high-sensitivity cardiac troponin I levels in patients with suspected acute coronary syndrome. JAMA Cardiol 2016;1:405-12.

14 Pickering JW, Than MP, Cullen L, et al. Rapid rule-out of acute myocardial infarction with a single high-sensitivity cardiac troponin $\mathrm{T}$ measurement below the limit of detection: a collaborative meta-analysis. Ann Intern Med 2017; 166:715-24.

15 Greenslade J, Cho E, Van Hise C, et al. Evaluating rapid rule-out of acute myocardial infarction using a high-sensitivity cardiac troponin I assay at presentation. Clin Chem 2018;64:820-9.

16 Body R, Mahler S. Welcome to the real world: do the conditions of FDA approval Devalue high-sensitivity troponin? Acad Emerg Med 2017;24:1278-80.
17 Roffi M, Patrono C, Collet J-P, et al. 2015 ESC guidelines for the management of acute coronary syndromes in patients presenting without persistent ST-segment elevation: Task force for the management of acute coronary syndromes in patients presenting without persistent ST-segment elevation of the European Society of cardiology (ESC). Eur Heart J 2016;37:267-315.

18 NICE guidance. Chest pain of recent onset: assessment and diagnosis (update). CG95. London: National Institute for Health and Care Excellence, 2016.

19 Thapa S, Wong R, Goodacre S. Implementation of rapid rule out of myocardial infarction using high-sensitivity troponin: cross-sectional survey of English hospitals. Emerg Med J 2020;37:229-31.

20 Carlton E, Campbell S, Ingram J, et al. Randomised controlled trial of the limit of detection of troponin and ECG discharge (LODED) strategy versus usual care in adult patients with chest pain attending the emergency department: study protocol. BMJ Open 2018;8:025339.

21 Than M, Aldous S, Lord SJ, et al. A 2-hour diagnostic protocol for possible cardiac chest pain in the emergency department: a randomized clinical trial. JAMA Intern Med 2014;174:51-8.

22 Reichlin T, Irfan A, Twerenbold R, et al. Utility of absolute and relative changes in cardiac troponin concentrations in the early diagnosis of acute myocardial infarction. Circulation 2011;124:136-45.

23 Chew DP, Lambrakis K, Blyth A, et al. A randomized trial of a 1-hour troponin T protocol in suspected acute coronary syndromes: the rapid assessment of possible acute coronary syndrome in the emergency department with high-sensitivity troponin T study (RAPID-TnT). Circulation 2019;140:1543-56.

24 Carlton EW, Khattab A, Greaves K. Identifying patients suitable for discharge after a Single-Presentation high-sensitivity troponin result: a comparison of five established risk scores and two high-sensitivity assays. Ann Emerg Med 2015;66:635-45.

25 Body R, Carlton E, Sperrin M, et al. Troponin-only Manchester acute coronary syndromes (T-MACS) decision aid: single biomarker re-derivation and external validation in three cohorts. Emerg Med J 2017;34:349-56.

26 Poldervaart JM, Reitsma JB, Backus BE, et al. Effect of using the HEART score in patients with chest pain in the emergency department: a stepped-wedge, cluster randomized trial. Ann Intern Med 2017;166:689-97.

27 Bardaji A, Bonet G, Carrasquer A, et al. Prognostic implications of detectable cardiac troponin I below the 99th percentile in patients admitted to an emergency department without acute coronary syndrome. Clin Chem Lab Med 2018;56:1954-61.

28 Fox KAA, Dabbous $\mathrm{OH}$, Goldberg RJ, et al. Prediction of risk of death and myocardial infarction in the six months after presentation with acute coronary syndrome: prospective multinational observational study (GRACE). BMJ 2006;333:1091

29 Antman EM, Cohen M, Bernink PJ, et al. The TIMI risk score for unstable angina/ non-ST elevation MI: a method for prognostication and therapeutic decision making. JAMA 2000;284:835-42. 